\title{
Erratum: Bifurcation threshold of the delayed van der Pol oscillator under stochastic modulation [Phys. Rev. E 85, 056214 (2012)]
}

\author{
Mathieu Gaudreault, François Drolet, and Jorge Viñals
}

(Received 27 November 2019; accepted 21 January 2020; published 11 February 2020)

DOI: 10.1103/PhysRevE.101.029901

The second-order differential equation [Eq. (1) in the original paper] has to be written as

$$
\begin{gathered}
\dot{x}(t)=y(t), \\
\dot{y}(t)+\omega_{0}^{2} x(t)+\eta x(t-\tau)=\beta y(t)+\kappa y(t-\tau)-b x^{2}(t) y(t)+x(t) \xi(t) .
\end{gathered}
$$

With the same expansion given in the original paper, Eq. (9) is replaced by

$$
\begin{aligned}
& \epsilon^{3} \partial_{T} A(T) \cos (\omega t)-\epsilon^{3} \partial_{T} B(T) \sin (\omega t)=0 \\
& \epsilon^{3} \partial_{T} A(T) \sin (\omega t)+\epsilon^{3} \partial_{T} B(T) \cos (\omega t)=\frac{1}{\omega}\{\mu[\epsilon A(T) \sin (\omega t)+\epsilon B(T) \cos (\omega t)] \\
& -b \omega[\epsilon A(T) \cos (\omega t)-\epsilon B(T) \sin (\omega t)]^{2}[\epsilon A(T) \sin (\omega t)+\epsilon B(T) \cos (\omega t)] \\
& -[\epsilon A(T) \cos (\omega t)-\epsilon B(T) \sin (\omega t)] \xi(t)\}
\end{aligned}
$$

Equations (16) and (17) are then replaced by

$$
\begin{aligned}
\epsilon^{3} \partial_{T} A(T)= & \frac{\sin (\omega t)}{\omega}\{\mu[\epsilon A(T) \sin (\omega t)+\epsilon B(T) \cos (\omega t)] \\
& -b \omega[\epsilon A(T) \cos (\omega t)-\epsilon B(T) \sin (\omega t)]^{2}[\epsilon A(T) \sin (\omega 7 t)+\epsilon B(T) \cos (\omega t)] \\
& -[\epsilon A(T) \cos (\omega t)-\epsilon B(T) \sin (\omega t)] \xi(t)\} \\
\epsilon^{3} \partial_{T} B(T)= & \frac{\cos (\omega t)}{\omega}\{\mu[\epsilon A(T) \sin (\omega t)+\epsilon B(T) \cos (\omega t)] \\
& -b \omega[\epsilon A(T) \cos (\omega t)-\epsilon B(T) \sin (\omega t)]^{2}[\epsilon A(T) \sin (\omega t)+\epsilon B(T) \cos (\omega t)] \\
& -[\epsilon A(T) \cos (\omega t)-\epsilon B(T) \sin (\omega t)] \xi(t)\}
\end{aligned}
$$

so that our final result [Eq. (18) in the original manuscript] reads

$$
\begin{aligned}
\frac{d}{d T}\left[\begin{array}{l}
A \\
B
\end{array}\right]= & \frac{1}{2 \omega}\left[\begin{array}{cc}
\tilde{\mu} & 0 \\
0 & \tilde{\mu}
\end{array}\right]\left[\begin{array}{l}
A \\
B
\end{array}\right]-\frac{b}{8}\left[\begin{array}{l}
A\left(A^{2}+B^{2}\right) \\
B\left(A^{2}+B^{2}\right)
\end{array}\right]+\frac{1}{2 \epsilon \omega}\left[\begin{array}{cc}
0 & 1 \\
-1 & 0
\end{array}\right]\left[\begin{array}{l}
A \\
B
\end{array}\right] \xi_{0}(T) \\
& +\frac{1}{2 \sqrt{2} \epsilon \omega}\left\{\left[\begin{array}{cc}
0 & -1 \\
-1 & 0
\end{array}\right]\left[\begin{array}{l}
A \\
B
\end{array}\right] \xi_{1}(T)+\left[\begin{array}{cc}
-1 & 0 \\
0 & 1
\end{array}\right]\left[\begin{array}{l}
A \\
B
\end{array}\right] \xi_{2}(T)\right\} .
\end{aligned}
$$

There is a global factor of $1 / 2$ in the right-hand side of Eq. (7) relative to Eq. (18) of the original paper. The different factor arises by having explicitly considered both Eqs. (1) and (2) in the expansion. As a consequence, Eq. (29) is replaced by

$$
\alpha=\frac{4 \omega \tilde{\mu}}{\tilde{D}}+1,
$$

and the bifurcation threshold is given by

$$
2\left(\omega_{0}^{2}+\eta\right)\left(\beta_{c}+\eta \tau+\kappa\right)=-D(1+\kappa \tau)
$$

There is an additional factor of 2 in the left-hand side of this equation compared to Eq. (32) of the original paper. 


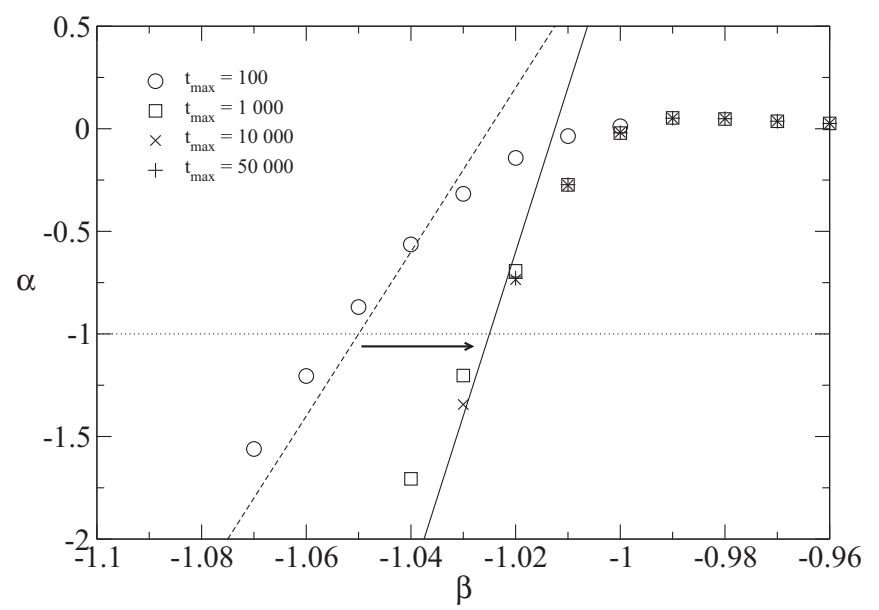

FIG. 1. Exponent of the power law $\alpha$ as a function of the damping parameter $\beta$ calculated from the stationary probability distribution function $p(x)$ of the position $x$ of the stochastic van der Pol oscillator with delayed feedback. The parameters are fixed at $\omega_{0}=1, \eta=1, b=1, \kappa=1, \tau=0$, and $D=0.1$. The calculations are done for different integration times $t_{\max }$. The bifurcation point is located at $\alpha=-1$, the point at which the distribution function becomes nonnormalizable. The symbols are the results of the numerical simulations whereas the dashed curve is the previous results in the original paper, and the solid curve is the new theoretical prediction, Eq. (8).

Unfortunately, the results of the numerical analysis of this model described in the original paper (Figs. 5 and 6) were based on short time runs that had not reached a stationary state. Therefore the agreement between theory and computation in that publication was spurious. We have recomputed the threshold value of $\alpha$, and the results are shown in Fig. 1. Our runs in Fig. 5 of the original paper considered integration up to dimensionless time $t_{\max }=100$. In Fig. 1, we show results for this value of $t_{\max }$, but also for much longer times $t_{\max }=1000,10000$, and 50000 . The longer times agree with

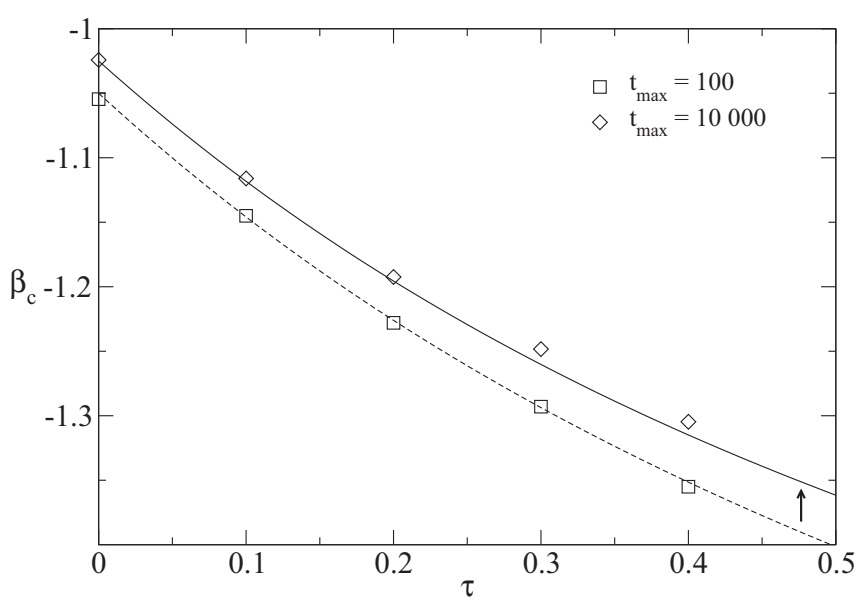

FIG. 2. Bifurcation diagram of Eq. (1). We show the critical value $\beta_{c}$ as a function of the time delay $\tau$. The other parameters are fixed at $\omega_{0}=1, \eta=1, b=1, \kappa=1$, and $D=0.1$. The symbols are the results of the numerical simulations for different integration times $t_{\max }$. The dashed curve is the previous results in the original paper, and the solid curve is the new theoretical prediction for the threshold location, Eq. (9).

each other, showing convergence, and agree with the analytic result of Eq. (9). Figure 2 amends Fig. 6 of the original paper and shows agreement between the corrected analytic threshold and the new numerical calculation.

The general analysis and conclusions of the original paper still stand. However, the location of the stability threshold was erroneous by a factor of two, fact that has been pointed out in Refs. [1,2]. The corrected result is now in agreement with Ref. [3] and with more recent research in Refs. [1] and [2] in the limit of no delay $(\tau=\kappa=\eta=0)$.

This research has been supported by the Extreme Science and Engineering Discovery Environment (XSEDE) [4], which is supported by National Science Foundation Grant No. ACI1548562 .
[1] A. A. Dubkov and I. A. Litovsky, Probabilistic characteristics of noisy Van der Pol-type oscillator with nonlinear damping, J. Stat. Mech.: Theor. Exp. (2016) 054036.

[2] N. Lingala and N. Sri Namachchivaya, Perturbations of linear delay differential equations at the verge of instability, Phys. Rev. E 93, 062104 (2016).
[3] C. Mayol, R. Toral, and C. R. Mirasso, Derivation of amplitude equations for nonlinear oscillators subject to arbitrary forcing, Phys. Rev. E 69, 066141 (2004).

[4] J. Towns, T. Cockerill, M. Dahan, I. Foster, K. Gaither, A. Grimshaw, V. Hazlewood, S. Lathrop, D. Lifka, G. D. Peterson et al., XSEDE: Accelerating scientific discovery, Comput. Sci. Eng. 16, 62 (2014). 